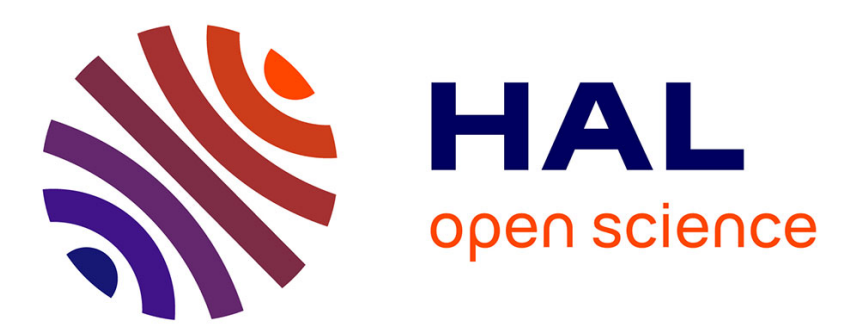

\title{
Mixed nonparametric-parametric probabilistic model for earthquake reliability of an inelastic reinforced concrete frame structure
}

\author{
Christophe Desceliers, Guy Bonnet, S. Hamza, P. Delmotte
}

\section{To cite this version:}

Christophe Desceliers, Guy Bonnet, S. Hamza, P. Delmotte. Mixed nonparametric-parametric probabilistic model for earthquake reliability of an inelastic reinforced concrete frame structure. Bulletin of Earthquake Engineering, 2010, 8 (4), pp.921-935. hal-00750183

\section{HAL Id: hal-00750183 \\ https://hal.science/hal-00750183}

Submitted on 14 Nov 2012

HAL is a multi-disciplinary open access archive for the deposit and dissemination of scientific research documents, whether they are published or not. The documents may come from teaching and research institutions in France or abroad, or from public or private research centers.
L'archive ouverte pluridisciplinaire HAL, est destinée au dépôt et à la diffusion de documents scientifiques de niveau recherche, publiés ou non, émanant des établissements d'enseignement et de recherche français ou étrangers, des laboratoires publics ou privés. 


\title{
Mixed nonparametric-parametric probabilistic model for earthquake reliability of an inelastic reinforced concrete frame structure
}

\author{
C. Desceliers • G. Bonnet • S. Hamza • \\ P. Delmotte
}

Received: date / Accepted: date

\begin{abstract}
The paper is devoted to the study of uncertainties when studying buildings under seismic loading. These uncertainties are related to the simplifications used when constructing the model (model uncertainties) and to the numerical data needed at the computation stage (data uncertainties). It has been shown in previous papers that nonparametric models are able, in the case of linear dynamics, to deal simultaneously with these two kinds of uncertainties. The paper presents an extension of this kind of model by taking into account a "mixed" approach for concrete frame structures, which uses a nonparametric model for the part of the structure which behaves linearly and a parametric approach for the parts of the structure (plastic hinges) which behave non-linearly. A numerical application is presented in the case of a residential building.
\end{abstract}

Keywords Probabilistic model · earthquake reliability · nonlinear dynamics · inelastic material

\section{Introduction}

The estimation of the response of existing buildings when submitted to seismic loads led to the use of pseudo-static methods and of fully non-linear dynamic formulations. In this last case, a mechanical model of the building has to be constructed to predict its transient nonlinear dynamical response. However, the position of the parts of the structure needing a nonlinear modelling is usually known, because it involves plastic hinges which are known from the engineer. For instance, in the case of a building with a reinforced concrete frame structure, the nonlinearities are related to the inelastic behaviour of the material at the ends of the beams and columns $[4,10]$. The complexity level of a

C. Desceliers, G. Bonnet, S. Hamza

Université Paris-Est, Modélisation et Simulation Multi Echelle, MSME FRE3160 CNRS, 5 bd Descartes, 77454 Marne-la-Vallée cedex 02, France

Tel.: +33-1-60-95-79

Fax: +33-1-60-95-99

E-mail: christophe.desceliers@univ-paris-est.fr, guy.bonnet@univ-paris-est.fr

P. Delmotte Centre Scientifique et Technique du Batiment, 77454, Marne-laVallée cedex 02, France 
real building is so high that modelling approximations are necessarily introduced, which yield a model built by the engineer called thereafter "simplified nonlinear mechanical model". Such a model can be improved by taking into account all the uncertainties through reliability analyses (see for instance $[6,11]$ ) and by considering that the simplified model is the "mean model" in the probabilistic sense. The sources of uncertainties are related to the data of the model (for instance, the mechanical parameters of the inelastic constitutive equations) and to the model approximations introduced when constructing the simplified nonlinear mechanichal model (for instance, suppression of "non-significant" structural elements). These uncertainties are very significant in the case of existing buildings due to the lack of data concerning the original design and the historical evolution of the mechanical properties of the materials (concrete damage, corrosion...).

In this paper, a residential building is considered for applying the proposed methodology. A simplified nonlinear mechanical model is built by using the finite element method. In this model, the reinforced concrete frame structure of the building is meshed and secondary parts are represented by their masses. The Takeda nonlinear model [18] is used to describe the inelastic constitutive equations of the material at the ends of the beams and columns of the frame structure. These inelastic constitutive equations are mainly relevant for lateral loads as induced by horizontal ground accelerations. A local-to-global approach [1] is used to compute the parameters of the inelastic constitutive equations at the macroscopic scale. Other parts of the structure is assumed to be made up of a linear viscoelastic medium. Then, a reduced nonlinear model is built by using the eigenvectors of the associated undamped linearized model and a stochastic model is developed to take into account the uncertainties by modelling (1) the parameters of the inelastic constitutive equations as random variables (parametric approach) and (2) the generalized mass, stiffness and damping matrices as random matrices (nonparametric approach). The probabilistic model of these random variables and random matrices are built by using the information theory (see for instance [7-9, $13,14,16]$ ). According to this theory, the entropy of a random system is a functional of the probability density function related to the uncertain physical parameters. The information theory states that each probability density function must be chosen as the one which maximises the entropy of the system with the constrains to comply with all known informations (mean values, standard deviations, etc). Thus, we present a mixed nonparametric-parametric probabilistic approach for modelling the uncertainties of a nonlinear dynamical system. Such a formulation has already been proposed for instance in $[2,15])$ in the case of geometric nonlinearities. In this paper, we propose to develop such a formulation for structures where nonlinearities are related to the elastoplastic behaviour of constitutive materials.

\section{Description of the structure and of the seismic motion}

The methodology developed in this paper is applied to a relatively simple existing residential building (see Fig.1). The building under consideration is a reinforced concrete frame structure. The strength of the structure is mainly due to the columns whose sections are between $15 \mathrm{~cm} \times 15 \mathrm{~cm}$ and $50 \mathrm{~cm} \times 15 \mathrm{~cm}$. It is assumed that the floor and the roof are made up of a linear homogeneous isotropic material and that the frames (beams and columns) are made up of an inelastic heterogenous anisotropic material. 
Let $(O, \mathbf{X}, \mathbf{Y}, \mathbf{Z})$ be an orthogonal reference frame such that $(O, \mathbf{X}, \mathbf{Y})$ is the horizontal plane of the structure. The structure is submitted to a seismic loading that induces a ground acceleration along direction $\mathbf{X}$ and for which the time history is shown in Fig. 2-a. The modulus of the Fourier transform of the acceleration is shown in Fig. 2-b. It can be seen that the accelerogram belongs to the frequency band $B_{1}=[0,50] \mathrm{Hz}$.

(a)

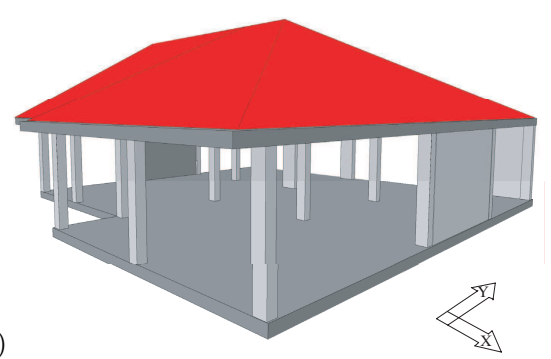

(b)

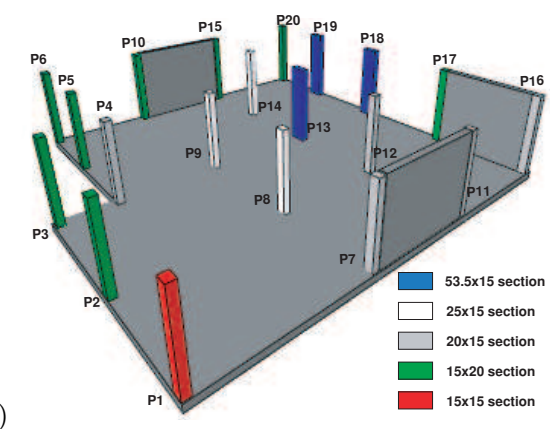

(c)

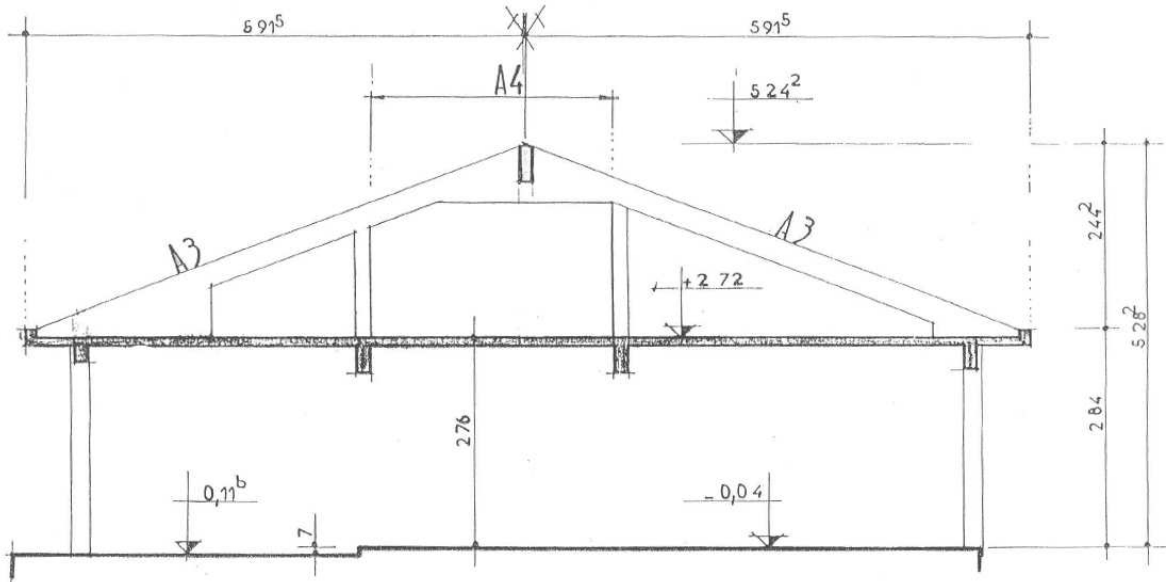

Fig. 1 Residential building under consideration (Fig. a), columns distribution (Fig. b) and a typical outline (Fig. c)

\section{Constitutive equations for the material which behaves inelastically}

As explained in the introduction, the inelastic behaviour of the material at the ends of the beams and columns of the reinforced concrete frame structure is strongly relevant for lateral loads induced by horizontal accelerations. Such a behaviour induces localized plastic hinges. The Takeda nonlinear model [18] is used to describe the inelastic constitutive equations within the hinges. The Takeda nonlinear model for a beam is completely defined by a trilinear moment-curvature curve, representing the elastic undamaged, damaged and post yielding stages [18]. This trilinear moment-curvature curve is defined by 6 mechanical parameters (see Fig. 3 -a): the curvature and moment 
(a)

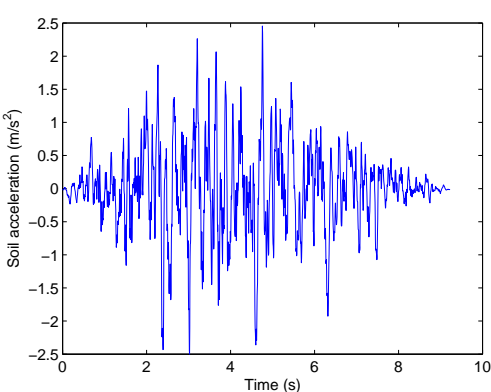

(b)

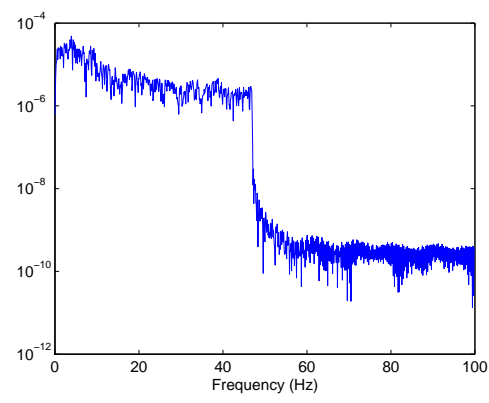

Fig. 2 Accelerogram of the seismic signal (Fig. a) and modulus of the Fourier transform of the seismic signal (Fig. b)

(a)

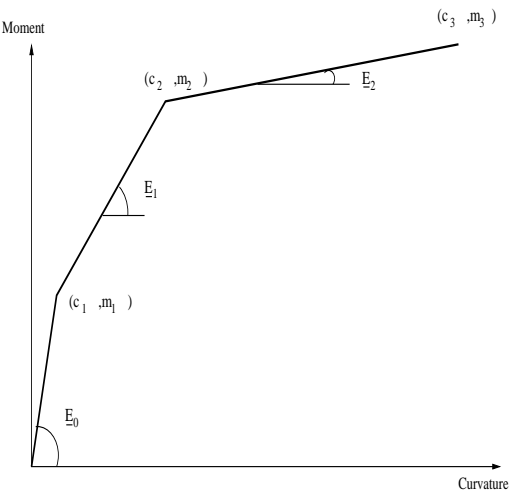

(b)

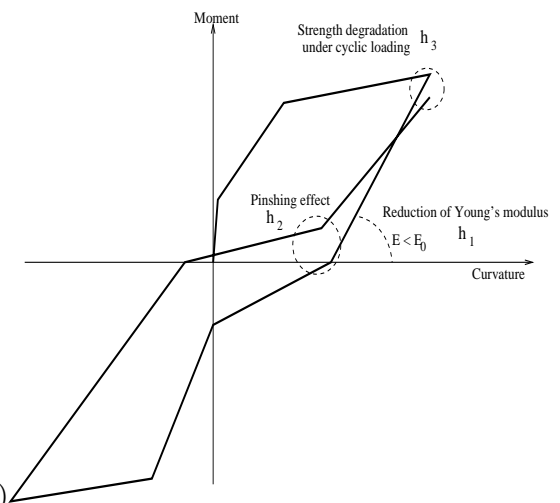

Fig. 3 (a) Takeda characteristic trilinear moment-curvature curve - (b) Moment-curvature curve under cyclic loading using Takeda nonlinear model

at the beginning of the damaged state, $c_{1}$ and $m_{1}$, the yielding curvature and moment $c_{2}$ and $m_{2}$ and the collapse curvature and moment $c_{3}$ and $m_{3}$. All these parameters are such that $0<c_{1} \leq c_{2} \leq c_{3}$ and $0<m_{1} \leq m_{2} \leq m_{3}$. In addition, the following equations hold

$$
\begin{array}{r}
m_{1}=\underline{E}_{0} \underline{I} c_{1} \\
m_{2}=m_{1}+\underline{E}_{1} \underline{I}\left(c_{2}-c_{1}\right), \\
m_{3}=m_{2}+\underline{E}_{2} \underline{I}\left(c_{3}-c_{2}\right),
\end{array}
$$

where $\underline{E}_{0}, \underline{E}_{1}, \underline{E}_{2}$ are the elastic, cracked and plastic stiffness moduli and $\underline{I}$ is the moment of inertia of the cross section of the beam ( underlined characters are used to avoid confusion with other notations within the paper). In addition, the hysteretic phenomena are controlled by 3 parameters: $h_{1}$ for the reduction of Young's modulus during cyclic loading, $h_{2}$ for the pinching effect and $h_{3}$ for the strength degradation under cyclic loading (see Fig. 3-b). 


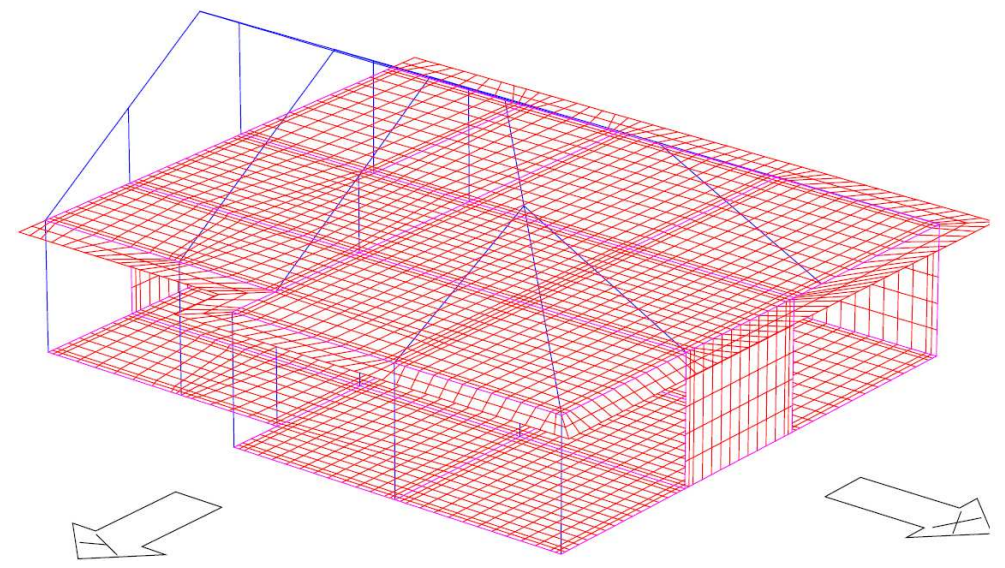

Fig. 4 The finite element mesh of the simplified nonlinear mechanical model

\section{Nonlinear mechanical model}

For such a structure, the simplified nonlinear mechanical model (see section 1) is built by using the finite element method (see for instance [19]) for the reinforced concrete frame structure. The finite element mesh used thereafter is shown in Fig. 3. It is made of 436 beam and plate elements and comprises 2006 degrees of freedom. Let $[M],[D]$ and $[K]$ be the finite element mass, damping and stiffness matrices of the reinforced concrete frame structure. At time $t>0$, the vector of the $N$ degrees of freedom related to the finite element mesh of the structure is denoted by $\mathbf{u}(t)$ and belongs to $\mathbb{R}^{N}$. We then have, for $t>0$

$$
[M] \ddot{\mathbf{u}}(t)+[D] \dot{\mathbf{u}}(t)+[K] \mathbf{u}(t)=-[M] \ddot{\mathbf{u}}_{s}(t)-\mathbf{g}_{N L}\left(\{\mathbf{u}(\tau)\}_{0 \leq \tau \leq t}, t ; \mathbf{w}\right) \quad,
$$

with the initial conditions

$$
\mathbf{u}(0)=\dot{\mathbf{u}}(0)=\ddot{\mathbf{u}}_{s}(0)=0,
$$

where a dot means the derivative with respect to time $t$ and $\mathbf{u}_{s}(t)$ is the vector of the constrained degrees of freedom at the foundation points of the structure meshing. Stiffness matrix $[K]$ takes into account the linear part of the elastoplastic model (modulus $\underline{E}_{0}$ shown in Fig. 3-a). Matrices $[M],[D],[K]$ define the low strain "linear model" used thereafter to build a basis of eigenvectors. The vector of the "equivalent nonlinear forces" $\mathbf{g}_{N L}$ represents the effect of the inelastic constitutive equations of the material at the ends of the beams and columns of the reinforced concrete frame structure. The vector $\mathbf{w}=\left(c_{1}, m_{1}, c_{2}, m_{2}, c_{3}, m_{3}, h_{1}, h_{2}, h_{3}\right)$ includes all parameters of the Takeda nonlinear model. It should be noted that the vector of the "nonlinear forces" $\mathbf{g}_{N L}$ depends of the parameters of the Takeda model (components of vector $\mathbf{w}$ ) and of the history $\{\mathbf{u}(\tau)\}_{0 \leq \tau \leq t}$ of $\mathbf{u}$.

\section{Modal formulation of the nonlinear model : the reduced nonlinear model}

The formulation of the previous section has shown that taking into account the nonlinear behaviour of the plastic hinges leads to solve a system of nonlinear equations. 
From another point of view, the nonparametric model is built from the reduced dynamic model of the structures built on the linear modes. A modal formulation of the nonlinear model, built on the modes related to the associated linear model is therefore presented in this section. Let $\lambda_{1} \leq \ldots \leq \lambda_{N}$ be the $N$ eigenvalues which are solutions of the generalized eigenvalue problem: find $\phi \in \mathbb{R}^{N}$ and $\lambda>0$ such that $[K] \phi=\lambda[M] \phi$. Let $\left\{\phi_{1}, \ldots, \phi_{N}\right\}$ be the $N$ eigenvectors associated with eigenvalues $\lambda_{1} \leq \ldots \leq \lambda_{N}$. Then, the vector $\mathbf{u}(t)$ can be rewritten as $\mathbf{u}(t)=\sum_{\alpha=1}^{N} q_{\alpha}(t) \phi_{\alpha}$ where $q_{\alpha}(t)$ are the generalized coordinates of vector $\mathbf{u}(t)$ within the basis of eigenvectors $\phi_{\alpha}$. The reduced nonlinear model is built with the approximation $\mathbf{u}(t) \simeq \mathbf{u}^{n}(t)$ in which

$$
\mathbf{u}^{n}(t)=\sum_{\alpha=1}^{n} q_{\alpha}(t) \phi_{\alpha},
$$

where $n$ is the order of the reduced modal expansion which is chosen such that $n<N$. This last equation is rewritten as

$$
\mathbf{u}^{n}(t)=\left[\Phi_{n}\right] \mathbf{q}^{n}(t)
$$

where the $n \times N$ modal matrix $\left[\Phi_{n}\right]$ is such that $\left[\Phi_{n}\right]_{\alpha j}=\left\{\phi_{\alpha}\right\}_{j}$ and where the vector $\mathbf{q}^{n}(t)$ is such that $\left\{\mathbf{q}^{n}(t)\right\}_{\alpha}=q_{\alpha}(t)$. Equations (4) and (5) yield

$$
\begin{gathered}
{[\mathcal{M}] \ddot{\mathbf{q}}^{n}(t)+[\mathcal{D}] \dot{\mathbf{q}}^{n}(t)+[\mathcal{K}] \mathbf{q}^{n}(t)=-[\mathcal{M}] \ddot{\mathbf{q}}_{\mathrm{s}}^{n}(t)-\left[\Phi_{n}\right]^{T} \mathbf{g}_{N L}\left(\left\{\left[\Phi_{n}\right] \mathbf{q}^{n}(\tau)\right\}_{0 \leq \tau \leq t}, t ; \mathbf{w}\right)} \\
\mathbf{q}^{n}(0)=\dot{\mathbf{q}}^{n}(0)=\ddot{\mathbf{q}}_{s}^{n}(0)=0
\end{gathered}
$$

where $[\mathcal{M}],[\mathcal{D}]$ and $[\mathcal{K}]$ are the generalized mass, damping and stiffness matrices defined as

$$
[\mathcal{M}]=\left[\Phi_{n}\right]^{T}[M]\left[\Phi_{n}\right] \quad, \quad[\mathcal{D}]=\left[\Phi_{n}\right]^{T}[D]\left[\Phi_{n}\right] \quad, \quad[\mathcal{K}]=\left[\Phi_{n}\right]^{T}[K]\left[\Phi_{n}\right],
$$

and where $\mathbf{q}_{s}^{n}=[\mathcal{M}]^{-1}\left[\Phi_{n}\right]^{T}[M] \mathbf{u}_{s}(t)$. The second term in the right hand side of Eq. (8) represents the "equivalent nonlinear forces" due to the nonlinearities induced by the inelastic behaviour of materials (see section 3 ). It should be noted that the nonlinear forces are located at the ends of the beams and columns within the frame structure. It is noteworthy that strong nonlinear deformations do appear within these parts of the structure and that a projection onto the low frequency eigenvectors only is not sufficient to represent accurately such a deformation. Then, it is necessary to use a basis which also contains higher frequencies eigenvectors that allow local displacements to be accurately represented even if they are out of the frequency content of the signal related to seismic loading. Thus, a higher number of eigenvectors may be necessary to build the reduced nonlinear model.

For any given symmetric positive-definite matrices $[\mathcal{M}],[\mathcal{D}],[\mathcal{K}]$ and any given vector w, Eqs. (8) and (9) are solved by using an implicit Newmark scheme. For each time step of the Newmark scheme, the nonlinear equations are solved with a Newton algorithm. Then, using Eq. (7), approximation $t \mapsto \mathbf{u}^{n}(t)$ can be built. Consequently, we can define a mapping $\mathbf{f}^{n}$ such that for any values of $[\mathcal{M}],[\mathcal{D}],[\mathcal{K}]$ and $\mathbf{w}$, displacement $\mathbf{u}^{n}(t)$ is given by

$$
\mathbf{u}^{n}(t)=\mathbf{f}^{n}(t ;[\mathcal{M}],[\mathcal{D}],[\mathcal{K}], \mathbf{w})
$$


6 Stochastic model

6.1 Mixed nonparametric-parametric probabilistic formulation of the uncertainties

This chapter is devoted to the presentation of the probabilistic approach which is used to account for the uncertainties involved during the process of studying the behaviour of buildings under seismic loading. From a general point of view, the designer prepares a "nominal model" which is the best approximation which takes into account the main features of the structure under study : geometry, structural elements, physical properties, etc, as defined within the previous sections. When a probabilistic approach is used, this "nominal model" can be considered as a "mean model" (in the probabilistic sense), within a probabilistic model whose objective is to take into account uncertainties related to the geometry, the physical parameters,etc.

\begin{tabular}{|lll|}
\hline$($ A) Nominal model & \\
\hline $\begin{array}{l}\text { Simplification of the structure : } \\
\text { keeping main structural elements, } \\
\text { beam and plates simplification } \\
\text { use of mean beam and plate thickness } \\
\text { mean physical properties }\end{array}$ & $\rightarrow$ Mean model \\
\hline (B) Alea on parameters & $\rightarrow$ & Parametric model \\
\hline $\begin{array}{l}\text { Random physical properties, } \\
\text { Dispersion on thickness,... }\end{array}$ & \\
\hline (C) Alea on the model & NonParametric model \\
\hline $\begin{array}{l}\text { Random stiffness, damping } \\
\text { and mass matrices } \\
\text { Coupling induced between } \\
\text { dynamical modes }\end{array}$ \\
\hline Alea on nonlinear properties (B) + on model and linear properties (C) \\
\hline Randomness on all parts of the model $\rightarrow$ & Mixed model \\
\hline
\end{tabular}

Table 1 : Comparison of the different kinds of modelling

Table 1 presents different probabilistic approaches which account for the scattering of variables around the "mean model". For the usual probabilistic approach or so-called "parametric probabilistic approach", only uncertainties related to the physical parameters (mechanical strength, geometrical properties of the structural elements, etc) are taken into account, while for a "nonparametric probabilistic approach", it is possible to take into account, within a global approach and in the dynamic case, the uncertainties related to the model and to the physical parameters. The "model uncertainties" come from the necessary simplifications involved during the process of modelling : suppression of "non significant" structural elements, discretization procedures, simplified structural elements (beams, plates, etc). As shown in previous works, one of the consequences of these model uncertainties leads to the coupling of the modes which are estimated from the "mean model". The "nonparametric" method is therefore well suited to deal with structures or parts of the structures which behave linearly. The present work combines the parametric and nonparametric approaches to recover the advantages of both methods : uncertainties related to the linear part of the dynamical system are modeled by 
a "nonparametric" probabilistic approach and uncertainties related to the nonlinear part of the dynamical system are modeled by a "parametric" probabilistic approach. The approach thus developed is called "mixed probabilistic formulation", to recall both components of the method. In the following, both "nonparametric" and "parametric" probabilistic formulations are presented.

6.2 Nonparametric probabilistic formulation

Entering into more details, the nonparametric probabilistic approach (see for instance( [13-15]) consists in substituting in Eq. (11) the matrices $[\mathcal{M}],[\mathcal{D}],[\mathcal{K}]$ by random matrices $[\mathbf{M}],[\mathbf{D}],[\mathbf{K}]$. Usually, these random matrices are full as shown in [16]. In the following, it will be assumed that only uncertainties affecting the eigenfrequencies around the frequency content of the excitation signal are relevant for the dynamic computation of the structure. Let $\phi_{1}, \ldots, \phi_{n_{\mathrm{d}}}$, be the eigenvectors whose eigenfrequencies are relevant for the dynamic computation. Then, matrices $[\mathcal{M}],[\mathcal{D}],[\mathcal{K}]$ can be rewritten as :

$$
[\mathcal{M}]=\left[\begin{array}{cc}
{\left[\mathcal{M}_{n_{\mathrm{d}}}\right]} & 0 \\
0 & {\left[\mathcal{M}_{n_{\mathrm{qs}}}\right]}
\end{array}\right],[\mathcal{D}]=\left[\begin{array}{cc}
{\left[\mathcal{D}_{n_{\mathrm{d}}}\right]} & 0 \\
0 & {\left[\mathcal{D}_{n_{\mathrm{qs}}}\right]}
\end{array}\right],[\mathcal{K}]=\left[\begin{array}{cc}
{\left[\mathcal{K}_{n_{\mathrm{d}}}\right]} & 0 \\
0 & {\left[\mathcal{K}_{n_{\mathrm{qs}}}\right]}
\end{array}\right]
$$

where $\left[\mathcal{M}_{n_{\mathrm{d}}}\right],\left[\mathcal{D}_{n_{\mathrm{d}}}\right]$ and $\left[\mathcal{K}_{n_{\mathrm{d}}}\right]$ are positive diagonal $\left(n_{\mathrm{d}} \times n_{\mathrm{d}}\right)$ matrices and where $\left[\mathcal{M}_{n_{\mathrm{qs}}}\right],\left[\mathcal{D}_{n_{\mathrm{qs}}}\right]$ and $\left[\mathcal{K}_{n_{\mathrm{qs}}}\right]$ are positive diagonal $\left(n_{\mathrm{qs}} \times n_{\mathrm{qs}}\right)$ matrices such that $n_{\mathrm{qs}}=$ $n-n_{\mathrm{d}}$.

In the present paper, the nonparametric probabilistic model for the model uncertainties yields random matrices $[\mathbf{M}],[\mathbf{D}],[\mathbf{K}]$ defined as

$$
[\mathbf{M}]=\left[\begin{array}{cc}
{\left[\mathbf{M}_{n_{\mathrm{d}}}\right]} & 0 \\
0 & {\left[\mathcal{M}_{n_{\mathrm{qs}}}\right]}
\end{array}\right],[\mathbf{D}]=\left[\begin{array}{cc}
{\left[\mathbf{D}_{n_{\mathrm{d}}}\right]} & 0 \\
0 & {\left[\mathcal{D}_{n_{\mathrm{qs}}}\right]}
\end{array}\right],[\mathbf{K}]=\left[\begin{array}{cc}
{\left[\mathbf{K}_{n_{\mathrm{d}}}\right]} & 0 \\
0 & {\left[\mathcal{K}_{n_{\mathrm{qs}}}\right]}
\end{array}\right]
$$

where $\left[\mathbf{M}_{n_{\mathrm{d}}}\right],\left[\mathbf{D}_{n_{\mathrm{d}}}\right]$ and $\left[\mathbf{K}_{n_{\mathrm{d}}}\right]$ are random full matrices whose probabilistic model is built in [16] by using the information theory. In other words, it means that the probability density functions which appear in the model maximize the entropy under the constraint to comply to the available information. This information is defined as follows :

(1) $\left[\mathbf{M}_{n_{\mathrm{d}}}\right],\left[\mathbf{D}_{n_{\mathrm{d}}}\right],\left[\mathbf{K}_{n_{\mathrm{d}}}\right]$ are second order random variables with values in the set $\mathbb{M}^{+}$ of all the $\left(n_{\mathrm{d}} \times n_{\mathrm{d}}\right)$ real symmetric positive-definite matrices;

(2) the mean values of these random matrices are $E\left\{\left[\mathbf{M}_{n_{\mathrm{d}}}\right]\right\}=\left[\mathcal{M}_{n_{\mathrm{d}}}\right], E\left\{\left[\mathbf{D}_{n_{\mathrm{d}}}\right]\right\}=$ $\left[\mathcal{D}_{n_{\mathrm{d}}}\right], E\left\{\left[\mathbf{K}_{n_{\mathrm{d}}}\right]\right\}=\left[\mathcal{K}_{n_{\mathrm{d}}}\right]$;

(3) the Frobenius norm of inverse matrices $\left[\mathbf{M}_{n_{\mathrm{d}}}\right]^{-1},\left[\mathbf{D}_{n_{\mathrm{d}}}\right]^{-1},\left[\mathbf{K}_{n_{\mathrm{d}}}\right]^{-1}$ are secondorder random variables.

The nonparametric probabilistic model of such random matrices is presented [16] and briefly recalled in Appendix A. It should be noticed that the statistical fluctuation of random matrices $[\mathbf{M}],[\mathbf{D}],[\mathbf{K}]$ in this probabilistic model are only controlled by three dispersion coefficients $\delta_{\mathbf{M}}, \delta_{\mathbf{D}}$ and $\delta_{\mathbf{K}}$ (see also Appendix A). This low number of parameters accounting for the dispersion is one of the advantages of the method. 
6.3 Parametric probabilistic formulation of the uncertainties for the parameters of the inelastic constitutive equation

The parametric probabilistic approach consists in substituting in Eq. (11) the deterministic vector $\mathbf{w}=\left(c_{1}, m_{1}, c_{2}, m_{2}, c_{3}, m_{3}, h_{1}, h_{2}, h_{3}\right)$ which contains the physical parameters defining the non-linear behaviour of plastic hinges by a random vector $\mathbf{W}=\left(C_{1}, M_{1}, C_{2}, M_{2}, C_{3}, M_{3}, H_{1}, H_{2}, H_{3}\right)$ in which $C_{1}, M_{1}, C_{2}, M_{2}, C_{3}, M_{3}$ are statistically dependent $\mathbb{R}$-valued random variables such that, as deduced from Eqs (1) to (3),

$$
\begin{gathered}
M_{1}=\underline{E}_{0} \underline{I} C_{1} \quad, \quad M_{2}=M_{1}+\underline{E}_{1} \underline{I}\left(C_{2}-C_{1}\right) \quad, \quad M_{3}=M_{2}+\underline{E}_{2} \underline{I}\left(C_{3}-C_{2}\right) \\
0<C_{1} \leq C_{2} \leq C_{3} \quad, \quad 0 \leq H_{1} \leq 1 \quad, \quad 0 \leq H_{2} \leq 1 \quad, \quad 0<H_{3}
\end{gathered}
$$

In addition, the mean values and the standard deviations of $C_{1}, C_{2}, C_{3}, H_{1}, H_{2}, H_{3}$ are assumed to be given and they are denoted by $c_{1}, c_{2}, c_{3}, h_{1}, h_{2}, h_{3}$ (for the mean values) and $\sigma_{C_{1}}, \sigma_{C_{2}}, \sigma_{C_{3}}, \sigma_{H_{1}}, \sigma_{H_{2}}, \sigma_{H_{3}}$ (for the standard deviations). The construction of the probabilistic model of these random variables is presented in [5] using the information theory and is briefly recalled in Appendix B.

\subsection{Random response spectrum}

The solution of the stochastic model is a $\mathbb{R}^{N}$-valued stochastic field $\left\{\mathbf{U}^{n}(t): t \in\right.$ $[0, T]\}$ such that

$$
\mathbf{U}^{n}(t)=\mathbf{f}^{n}(t ;[\mathbf{M}],[\mathbf{D}],[\mathbf{K}], \mathbf{W})
$$

It means that at each realization of $\mathbf{W},[\mathbf{M}],[\mathbf{D}],[\mathbf{K}]$ is associated a realization of $\mathbf{U}^{n}(t)$. Let $S_{j}(\xi, \omega)$ be the random normalized acceleration response spectrum of the stochastic transient response $\left\{\mathbf{U}^{n}\right\}_{j}$ of the $j$ th degree of freedom in which the damping ratio $\xi \in[0,1]$ and the eigenfrequency $\omega$ belongs to the frequency band of analysis $\left[\omega_{\min }, \omega_{\max }\right]$. We then have, for any given damping ratio $\xi$ and radial frequency $\omega$

$$
S_{j}(\xi, \omega)=\frac{\omega^{2}}{g} \max _{t \in[0, T]}\left|X_{j}(t)\right|
$$

where $g$ is a normalization constant and $X_{j}(t)$ is the $\mathbb{R}$-valued stochastic field indexed by $[0, T]$ such that

$$
\begin{array}{r}
\ddot{X}_{j}(t)+2 \xi \omega \dot{X}_{j}(t)+\omega^{2} X_{j}(t)=-\left\{\ddot{\mathbf{U}}^{n}(t)\right\}_{j}, t \in[0, T], \\
X_{j}(0)=\dot{X}_{j}(0)=0 .
\end{array}
$$

The stochastic solver used to estimate the confidence region of $S_{j}(\xi, \omega)$ is based on the Monte Carlo method and is presented in Appendix C. 


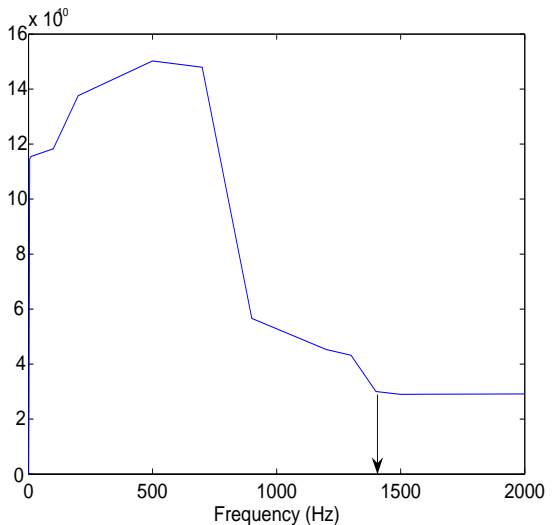

Fig. 5 Convergence analysis of the reduced matrix model. Graph of the mapping $n \mapsto$ $\operatorname{Conv}_{1}(n)$. Horizontal axis: dimension $n$ of the reduced model. Vertical axis: $\operatorname{Conv}_{1}(n)$

\section{Application and numerical results}

\subsection{Parameters of the stochastic model for the application}

In this section, the results related to an application concerning the building defined in section 2 are presented. For this application, it is necessary to precise all parameters defining the probabilistic model : mean values and dispersion parameters in the case of the parametric part of the model(parameters related to the nonlinear behaviour), dispersion parameters in the case of the nonparametric part of the model (dispersion parameters on stiffness, damping and mass matrices). The application is performed hereafter with $\delta_{M}=\delta_{D}=\delta_{K}=0.15$ for the nonparametric probabilistic formulation and $c_{1}=0.0018 \mathrm{~m}^{-1}, m_{1}=10.5 \times 10^{3} \mathrm{~N} . \mathrm{m}, c_{2}=0.0123 \mathrm{~m}^{-1}, m_{2}=22 \times 10^{3} \mathrm{~N} . \mathrm{m}, c_{3}=$ $0.1225 \mathrm{~m}^{-1}, m_{3}=24.5 \times 10^{3} \mathrm{~N} . \mathrm{m}, h_{1}=0.3, h_{2}=0.8, h_{3}=50$ and the standard deviation $\sigma_{C_{1}}=9 \times 10^{-5} \mathrm{~m}^{-1}, \sigma_{C_{2}}=5 \times 10^{-3} \mathrm{~m}^{-1}, \sigma_{C_{3}}=6.13 \times 10^{-3} \mathrm{~m}^{-1}, \sigma_{H_{1}}=0.06$, $\sigma_{H_{2}}=0.23, \sigma_{H_{3}}=5$ for the parametric probabilistic formulation.

\subsubsection{Convergence analysis with respect to the number of modes $n$}

The convergence analysis of the nonlinear reduced model with respect to the parameter $n$ is carried out by using the mapping $n \mapsto \operatorname{Conv}_{1}(n)$ that is defined as

$$
\operatorname{Conv}_{1}(n)=\int_{0}^{T}\left\|\mathbf{t}\left(\mathbf{u}^{n}(t)\right)\right\|^{2} d t
$$

where $\mathbf{t}\left(\mathbf{u}^{n}(t)\right)$ is the vector of the shear forces due to the displacement $\mathbf{u}^{n}(t)=$ $\mathbf{f}^{n}(t ;[\mathcal{M}],[\mathcal{D}],[\mathcal{K}], \mathbf{w})$. Figure 5 shows the graph of mapping $n \mapsto \operatorname{Conv}_{1}(n)$. It can be deduced that the reduced nonlinear model is converged with $n=1400$ as shown by the plateau which can be seen in this figure. This number is high when compared with the number of eigenfrequenies belonging to the frequency band of analysis $B_{1}=[0,50] \mathrm{Hz}$ which is $n_{B_{1}}=5$. This high value of $n$ was discussed in section 5 . Figures 6 and 7 allow the solutions of the nonlinear model by time-step computation (i.e solving Eqs. (4 for the whole structure) and (5)) and of the reduced nonlinear model (i.e solving Eqs. (8) 

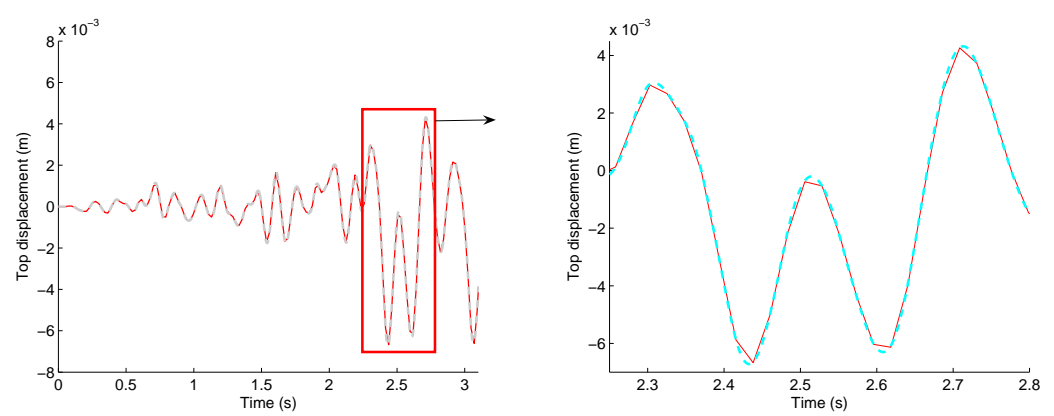

Fig. 6 Comparison between the time-step solution and the reduced modal solution. Graphs of the $j_{A}$ th degree of freedom $t \mapsto\{\mathbf{u}(t)\}_{j_{A}}$ (solid lines) and $t \mapsto\left\{\mathbf{u}^{n}(t)\right\}_{j_{A}}$ (dashed lines). Horizontal axis: time $t \in[0,3] \mathrm{s}$ (left) and time $t \in[2.3,2.8] \mathrm{s}$ (right). Vertical axis : $\{\mathbf{u}(t)\}_{j_{A}}$ (solid lines) and $\left\{\mathbf{u}^{n}(t)\right\}_{j_{A}}$ (dashed lines) in $m$
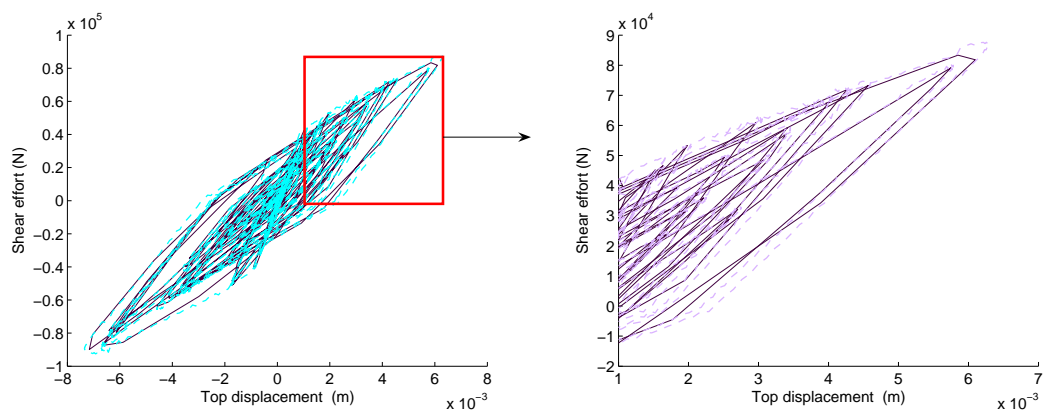

Fig. 7 Comparison between the time-step solution and the reduced modal solution. Graphs of the time evolution of the shear force $\{\mathbf{t}\}_{j_{A}}$ with respect to the displacements $\{\mathbf{u}(t)\}_{j_{A}}$ (solid lines) and $\left\{\mathbf{u}^{n}(t)\right\}_{j_{A}}$ (dashed lines). Horizontal axis: displacement $\{\mathbf{u}(t)\}_{j_{A}}$ and $\left\{\mathbf{u}^{n}(t)\right\}_{j_{A}}$ between $-8 \times 10^{-3} \mathrm{~m}$ and $8 \times 10^{-3} \mathrm{~m}$ (left) and between $\times 10^{-3} \mathrm{~m}$ and $6 \times 10^{-3} \mathrm{~m}$ (right). Vertical axis: $\{\mathbf{t}(\mathbf{u}(t))\}_{j_{A}}$ (solid lines) and $\left\{\mathbf{t}\left(\mathbf{u}^{n}(t)\right)\right\}_{j_{A}}$ (dashed lines) in $N$

and (9) and using Eq. (7)) to be compared. Figures 6 show the graphs of $t \mapsto\{\mathbf{u}(t)\}_{j_{A}}$ (solid lines) and $t \mapsto\left\{\mathbf{u}^{n}(t)\right\}_{j_{A}}$ (dashed lines) representing the displacement along direction $\mathbf{X}$ of the node $A$. Figures 7 show the time evolution of the shear forces $\{\mathbf{t}(\mathbf{u}(t))\}_{j_{A}}$ and $\left\{\mathbf{t}\left(\mathbf{u}^{n}(t)\right)\right\}_{j_{A}}$ at node $A$ with respect to the displacement $\{\mathbf{u}(t)\}_{j_{A}}$ (solid lines) and $\left\{\mathbf{u}^{n}(t)\right\}_{j_{A}}$ (dashed lines). It should be noted that, for node $A$ and $n=1400$, there is a good matching for displacements and shear forces obtained from the modal response and from the finite element computation taking into account all the structure and discretized time history.

\subsection{Confidence region of $S_{j}(\xi, \omega)$}

In order to buildthe confidence regions associated with a probability level $P_{c}=95 \%$ of the random normalized response spectrum, we choose $n_{\mathrm{d}}=n_{B_{2}}$ where $n_{B_{2}}=27$ is the number of eigenfrequencies that belong to the frequency band $B_{2}=[0,100] \mathrm{Hz}$. The stochastic solver used for this construction is based on the Monte Carlo method with $n_{\mathrm{S}}=300$ statistical independant realizations of $S_{j_{A_{k}}}(\xi, \omega)$ related to the displacement 

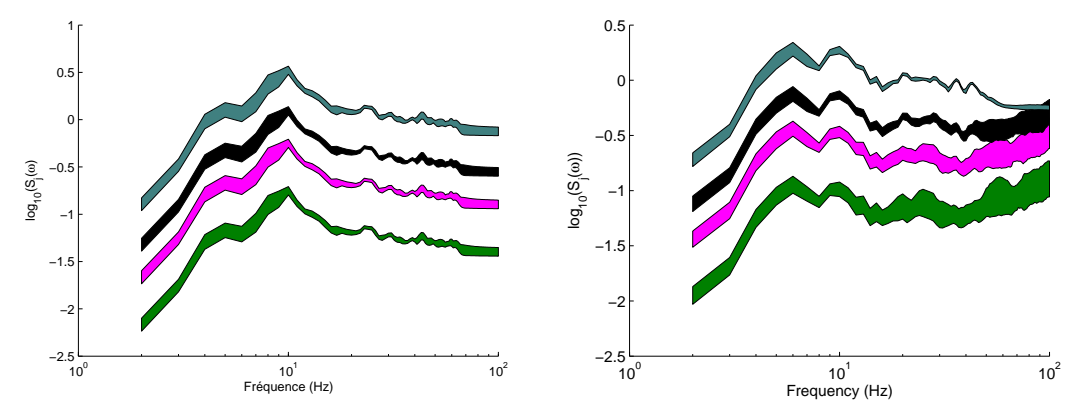

Fig. 8 Uncertainties quantification of the building for a linear stochastic approach (left) and a stochastic nonlinear approach (right). Horizontal axis: frequency in Hz. Vertical axis: confidence region of random acceleration spectrum $S_{j_{k}}(\xi, \omega)$ with the probability level $P_{c}=P_{c}=95 \%$ for the nodes $A_{k}$ with $k=1, \ldots, 4$ in the column $P_{13}$ (see fig. 1).

of node $A_{k}$ along direction $\mathbf{X}$ (see Fig. 1b) and whose coordinates are $\left(x_{k}, y_{k}, z_{k}\right)$ with $x_{k}=-6.93 \mathrm{~m}, y_{k}=6.3 \mathrm{~m}$ and $z_{k} \in[0,2.36] \mathrm{m}$.

The confidence regions of $S_{j}(\xi, \omega)$ for $j=j_{A_{1}}, j_{A_{2}}, j_{A_{3}}, j_{A_{4}}$, where $z_{1}=0.29 \mathrm{~m}$, $z_{2}=0.58 \mathrm{~m}, z_{3}=0.98 \mathrm{~m}$ and $z_{4}=2.36 \mathrm{~m}$, are shown in Fig. 8 (right) for the solution of the nonlinear model. It can be seen that for the frequency band $[0,10] \mathrm{Hz}$, the widthes of the confidence regions are nearly constant and similar for every node $A_{k}$. It means that the uncertainties levels related to the random acceleration response spectrum $S_{j_{A_{k}}}(\xi, \omega)$ do not depend of frequency $\omega$ and of coordinate $z_{k}$ of node $A_{k}$. On the other hand, the width of the confidence region in the frequency band $[10,100] \mathrm{Hz}$ increases with frequency $\omega$ and decreases with coordinate $z_{k}$. It means that the uncertainties levels increase with $\omega$ in frequency band $[10,100] \mathrm{Hz}$ and decrease with coordinate $z_{k}$. Thus, the most important uncertainties levels are reached for nodes close to the base of columns where plastic hinges are located. These plastic hinges support the most important plastic strain.

The confidence regions of $S_{j}(\xi, \omega)$ for $j=j_{A_{1}}, j_{A_{2}}, j_{A_{3}}, j_{A_{4}}, j_{A_{5}}$ are shown in Fig. 8 (left) for the linear case, i.e. with $\mathbf{g}_{N L}=0$ (stochastic linearized reduced model). It can be seen that the widthes of the confidence regions are similar for all nodes $A_{k}$ and for all $\omega$ in the frequency band of analysis. It means that the uncertainties levels are equivalent for all nodes $A_{k}$ and are nearly frequency independent in the frequency range $[0,10] \mathrm{Hz}$.

\section{Conclusions}

A mixed nonparametric-parametric probabilistic model for the uncertainties in a structure made up of inelastic materials has been presented. The nonparametric formulation allows the model uncertainties due to approximations in the construction of the simplified nonlinear model to be taken into account. The parametric formulation allows uncertainties related to the parameters of the inelastic materials to be taken into account. It should be noted that such a probablistic model does not allow to take into account uncertainties related to the seismic loads, physical nonlinearities other than those already accounted for, geometrical nonlinearities, etc. In addition, the main difficulty for using such a probabilistic model comes from the choice of the value of the 
dispersion parameters $\delta_{M}, \delta_{D}, \delta_{K}$. In other engineering fields, these parameters can be identified from experimental data by solving a stochastic inverse problem (see for instance $[3,17]$ ). Unfortunately, such experimental data are difficult to obtain in seismic engineering. In this case, these parameters must be chosen from expert appraisement. The parametric probabilistic model has been developed and presented in the paper using the information theory. The method developed in the paper allows the confidence regions of the random normalized acceleration response spectrum to be estimated by using a stochastic solver based on the Monte Carlo method. An example of one-storey building has been studied and it has been shown that material nonlinearities increase the uncertainties levels of the random normalized acceleration response spectrum when compared to the stochastic dynamical linear model, especially for frequencies higher than $10 \mathrm{~Hz}$ and for nodes located close to the base of the columns. Within the class of uncertainties taken into account by this probabilistic model, it appears that the nonlinear mean model is robust (no increase dispersion with frequency) up to frequencies around $20 \mathrm{~Hz}$ which is the practical upper band for frequency range in seismic engineering. In addition, the dispersion of sprectra would appear of second order compared to uncertainties associated with seismic excitations.

\section{A. Probabilistic model of random matrices for the nonparametric proba- bilistic formulation of the model uncertainties}

In this appendix, we present the construction of the probabilistic model of the random matrices involved by the nonparametric probabilistic formulation of the model uncertainties. It has been shown in [16] that the probabilistic model of random matrices $\left[\mathbf{M}_{n_{\mathrm{d}}}\right],\left[\mathbf{D}_{n_{\mathrm{d}}}\right]$ and $\left[\mathbf{K}_{n_{\mathrm{d}}}\right]$ can be constructed using the information theory with the available information (see section 6.2) yielding

$$
\left[\mathbf{M}_{n_{\mathrm{d}}}\right]=\left[\mathcal{L}_{\mathbf{M}}\right]^{T}\left[\mathbf{G}_{\mathbf{M}}\right]\left[\mathcal{L}_{\mathbf{M}}\right] \quad, \quad\left[\mathbf{D}_{n_{\mathrm{d}}}\right]=\left[\mathcal{L}_{\mathbf{D}}\right]^{T}\left[\mathbf{G}_{\mathbf{D}}\right]\left[\mathcal{L}_{\mathbf{D}}\right] \quad, \quad\left[\mathbf{K}_{n_{\mathrm{d}}}\right]=\left[\mathcal{L}_{\mathbf{K}}\right]^{T}\left[\mathbf{G}_{\mathbf{K}}\right]\left[\mathcal{L}_{\mathbf{K}}\right]
$$

in which the $\left(n_{\mathrm{d}} \times n_{\mathrm{d}}\right)$ upper triangular matrices $\left[\mathcal{L}_{\mathbf{M}}\right],\left[\mathcal{L}_{\mathbf{D}}\right],\left[\mathcal{L}_{\mathbf{K}}\right]$ correspond to the Cholesky factorizations $\left[\mathcal{M}_{n_{\mathrm{d}}}\right]=\left[\mathcal{L}_{\mathbf{M}}\right]^{T}\left[\mathcal{L}_{\mathbf{M}}\right],\left[\mathcal{D}_{n_{\mathrm{d}}}\right]=\left[\mathcal{L}_{\mathbf{D}}\right]^{T}\left[\mathcal{L}_{\mathbf{D}}\right],\left[\mathcal{K}_{n_{\mathrm{d}}}\right]=\left[\mathcal{L}_{\mathbf{K}}\right]^{T}\left[\mathcal{L}_{\mathbf{K}}\right]$ and where $\left[\mathbf{G}_{\mathbf{M}}\right],\left[\mathbf{G}_{\mathbf{D}}\right],\left[\mathbf{G}_{\mathbf{K}}\right]$ are random matrices for which the probability density functions $p_{\left[\mathbf{G}_{\mathbf{M}}\right]}$, $p_{\left[\mathbf{G}_{\mathbf{D}}\right]}, p_{\left[\mathbf{G}_{\mathbf{K}}\right]}$ are such that

$$
\begin{gathered}
p_{\left[\mathbf{G}_{\mathbf{M}}\right]}([G])=\mathbb{I}_{\mathbb{M}^{+}}([G]) c\left(\delta_{\mathbf{M}}\right)(\operatorname{det}[G])^{b\left(\delta_{\mathbf{M}}\right)} \exp \left\{-a\left(\delta_{\mathbf{M}}\right) \operatorname{tr}[G]\right\}, \\
p_{\left[\mathbf{G}_{\mathbf{D}]}\right.}([G])=\mathbb{I}_{\mathbb{M}^{+}}([G]) c\left(\delta_{\mathbf{D}}\right)(\operatorname{det}[G])^{b\left(\delta_{\mathbf{D}}\right)} \exp \left\{-a\left(\delta_{\mathbf{D}}\right) \operatorname{tr}[G]\right\}, \\
p_{\left[\mathbf{G}_{\mathbf{K}}\right]}([G])=\mathbb{I}_{\mathbb{M}^{+}}([G]) c\left(\delta_{\mathbf{K}}\right)(\operatorname{det}[G])^{b\left(\delta_{\mathbf{K}}\right)} \exp \left\{-a\left(\delta_{\mathbf{K}}\right) \operatorname{tr}[G]\right\},
\end{gathered}
$$

where $\mathbb{I}_{\mathbb{M}^{+}}([G])$ is equal to 1 if $[G]$ belongs to $\mathbb{M}^{+}$and is equal to zero if $[G]$ does not belong to $\mathbb{M}^{+}, \operatorname{tr}[G]$ is the trace of matrix $[G], a(\delta)=\left(n_{\mathrm{d}}+1\right) /\left(2 \delta^{2}\right), b(\delta)=a(\delta) \times\left(1-\delta^{2}\right), c(\delta)=$ $(2 \pi)^{-n_{\mathrm{d}}\left(n_{\mathrm{d}}-1\right) / 4} a(\delta)^{n_{\mathrm{d}} a(\delta)} / \prod_{j=1}^{n+1} \Gamma\left(\alpha_{j}(\delta)\right)$ in which $\alpha_{j}(\delta)=a(\delta)+(1-j) / 2$ and where $\Gamma$ is the Gamma function.

Then, it has then shown in $[16]$ that random matrices $\left[\mathbf{G}_{\mathbf{M}}\right],\left[\mathbf{G}_{\mathbf{D}}\right],\left[\mathbf{G}_{\mathbf{K}}\right]$ can be written

$$
\left[\mathbf{G}_{\mathbf{M}}\right]=\left[\mathbf{L}_{\mathbf{M}}\right]^{T}\left[\mathbf{L}_{\mathbf{M}}\right] \quad, \quad\left[\mathbf{G}_{\mathbf{D}}\right]=\left[\mathbf{L}_{\mathbf{D}}\right]^{T}\left[\mathbf{L}_{\mathbf{D}}\right] \quad, \quad\left[\mathbf{G}_{\mathbf{K}}\right]=\left[\mathbf{L}_{\mathbf{K}}\right]^{T}\left[\mathbf{L}_{\mathbf{K}}\right],
$$

where $\left[\mathbf{L}_{\mathbf{M}}\right]$, $\left[\mathbf{L}_{\mathbf{D}}\right]$ and $\left[\mathbf{L}_{\mathbf{K}}\right]$ are random upper triangular $\left(n_{\mathrm{d}} \times n_{\mathrm{d}}\right)$ real matrix such that

(1) for $j<j^{\prime},\left[\mathbf{L}_{\mathbf{M}}\right]_{j j^{\prime}},\left[\mathbf{L}_{\mathbf{D}}\right]_{j j^{\prime}}$ and $\left[\mathbf{L}_{\mathbf{K}}\right]_{j j^{\prime}}$ are real-valued Gaussian random variables with zero mean and variance equal to $\sigma_{\mathbf{M}}=\delta_{\mathbf{M}} / \sqrt{n_{\mathrm{d}}+1}, \sigma_{\mathbf{D}}=\delta_{\mathbf{D}} / \sqrt{n_{\mathrm{d}}+1}$ and $\sigma_{\mathbf{K}}=$ $\delta_{\mathbf{K}} / \sqrt{n_{\mathrm{d}}+1}$; 
(2) for $j=j^{\prime},\left[\mathbf{L}_{\mathbf{M}}\right]_{j j^{\prime}},\left[\mathbf{L}_{\mathbf{D}}\right]_{j j^{\prime}}$ and $\left[\mathbf{L}_{\mathbf{K}}\right]_{j j^{\prime}}$ are positive-valued random variables written as $\left[\mathbf{L}_{\mathbf{M}}\right]_{j j^{\prime}}=\sigma_{\mathbf{M}} \sqrt{2 V_{\mathbf{M}, j}},\left[\mathbf{L}_{\mathbf{D}}\right]_{j j^{\prime}}=\sigma_{\mathbf{D}} \sqrt{2 V_{\mathbf{D}, j}}$ where $V_{\mathbf{M}, j}, V_{\mathbf{D}, j}$ and $V_{\mathbf{K}, j}$ are positivevalued Gamma random variables whose probability density function $p_{V_{\mathbf{M}, j}}, p_{V_{\mathbf{D}, j}}, p_{V_{\mathbf{K}, j}}$ are written as

$p_{V_{\mathbf{M}, j}}(v)=\mathbb{I}_{\mathbb{R}^{+}}(v) \frac{v^{\alpha_{j}\left(\delta_{\mathbf{M}}\right)} e^{-v}}{\Gamma\left(\alpha_{j}\left(\delta_{\mathbf{M}}\right)\right.}, p_{V_{\mathbf{D}, j}}(v)=\mathbb{I}_{\mathbb{R}^{+}}(v) \frac{v^{\alpha_{j}\left(\delta_{\mathbf{D}}\right)} e^{-v}}{\Gamma\left(\alpha_{j}\left(\delta_{\mathbf{D}}\right)\right.}, p_{V_{\mathbf{K}, j}}(v)=\mathbb{I}_{\mathbb{R}^{+}}(v) \frac{v^{\alpha_{j}\left(\delta_{\mathbf{K}}\right)} e^{-v}}{\Gamma\left(\alpha_{j}\left(\delta_{\mathbf{K}}\right)\right.}$.

Then, $n_{\mathrm{s}}$ statistical independent realizations of random matrices $\left[\mathbf{M}_{n_{\mathrm{d}}}\right],\left[\mathbf{D}_{n_{\mathrm{d}}}\right]$ and $\left[\mathbf{K}_{n_{\mathrm{d}}}\right]$ are constructed such that

$$
\begin{gathered}
{\left[\mathbf{M}_{n_{\mathrm{d}}}\left(\theta_{1}\right)\right]=\left[\mathcal{L}_{\mathbf{M}}\right]^{T}\left[\mathbf{G}_{\mathbf{M}}\left(\theta_{1}\right)\right]\left[\mathcal{L}_{\mathbf{M}}\right], \ldots,\left[\mathbf{M}_{n_{\mathrm{d}}}\left(\theta_{n_{\mathrm{s}}}\right)\right]=\left[\mathcal{L}_{\mathbf{M}}\right]^{T}\left[\mathbf{G}_{\mathbf{M}}\left(\theta_{n_{\mathrm{s}}}\right)\right]\left[\mathcal{L}_{\mathbf{M}}\right]} \\
{\left[\mathbf{D}_{n_{\mathrm{d}}}\left(\theta_{1}\right)\right]=\left[\mathcal{L}_{\mathbf{D}}\right]^{T}\left[\mathbf{G}_{\mathbf{D}}\left(\theta_{1}\right)\right]\left[\mathcal{L}_{\mathbf{D}}\right], \ldots,\left[\mathbf{D}_{n_{\mathrm{d}}}\left(\theta_{n_{\mathrm{s}}}\right)\right]=\left[\mathcal{L}_{\mathbf{D}}\right]^{T}\left[\mathbf{G}_{\mathbf{D}}\left(\theta_{n_{\mathrm{s}}}\right)\right]\left[\mathcal{L}_{\mathbf{D}}\right]} \\
{\left[\mathbf{K}_{n_{\mathrm{d}}}\left(\theta_{1}\right)\right]=\left[\mathcal{L}_{\mathbf{K}}\right]^{T}\left[\mathbf{G}_{\mathbf{K}}\left(\theta_{1}\right)\right]\left[\mathcal{L}_{\mathbf{K}}\right], \ldots,\left[\mathbf{K}_{n_{\mathrm{d}}}\left(\theta_{n_{\mathrm{s}}}\right)\right]=\left[\mathcal{L}_{\mathbf{K}}\right]^{T}\left[\mathbf{G}_{\mathbf{K}}\left(\theta_{n_{\mathrm{s}}}\right)\right]\left[\mathcal{L}_{\mathbf{K}}\right]}
\end{gathered}
$$

B.Probabilistic model of the random vector for parametric formulation of uncertainties

related to the inelastic constitutive equations

In order to satisfy the first inequality in Eq. (15), random variables $C_{1}$ and $C_{2}$ are rewritten as

$$
C_{2}=A C_{1} \quad, \quad C_{3}=A B C_{1} \quad,
$$

where $A$ and $B$ are two random variables with values in $[1,+\infty[$ for which the mean values are $A=c_{2} / c_{1}$ and $B=c_{3} / c_{2}$ and for which the standard deviations $\sigma_{A}$ and $\sigma_{B}$ are such as (see [5]) $\sigma_{A}^{2}=\left(\sigma_{C_{2}}^{2}+c_{2}^{2}\right) /\left(\sigma_{C_{1}}^{2}+c_{1}^{2}\right)-\left(c_{2} / c_{1}\right)^{2}$ and $\sigma_{B}^{2}=\left(\sigma_{C_{3}}^{2}+c_{3}^{2}\right) /\left(\sigma_{C_{2}}^{2}+c_{2}^{2}\right)-\left(c_{3} / c_{2}\right)^{2}$. It should be noted that $\sigma_{A}$ and $\sigma_{B}$ are defined only if inequalities $\sigma_{C_{3}} / c_{3}>\sigma_{C_{2}} / c_{2}>\sigma_{C_{1}} / c_{1}$ hold. Let $p_{C_{1}}, p_{A}, p_{B}, p_{H_{1}}, p_{H_{2}}$ and $p_{H_{3}}$ be the probability density functions of random variables $C_{1}, A, B, H_{1}, H_{2}$ and $H_{3}$. It has been shown in [5] that

$$
\begin{array}{cccc}
p_{C_{1}}(x)=p_{1}\left(x ; c_{1}, \sigma_{C_{1}}, 0\right) & , \quad p_{A}(x)=p_{1}\left(x ; \underline{A}, \sigma_{A}, 1\right) \quad, \quad p_{B}(x)=p_{1}\left(x ; \underline{B}, \sigma_{B}, 1\right) \\
p_{H_{1}}(x)=p_{2}\left(x ; h_{1}, \sigma_{H_{1}}\right) \quad, & p_{H_{2}}(x)=p_{2}\left(x ; h_{2}, \sigma_{H_{2}}\right) \quad, \quad p_{H_{3}}(x)=p_{1}\left(x ; h_{3}, \sigma_{H_{3}}, 0\right)
\end{array}
$$

in which

$$
\begin{array}{r}
p_{1}(x ; \underline{X}, \sigma, a)=\mathbb{I}_{[a,+\infty[}(x) e^{-\sum_{k=1}^{3}\left\{\mathbf{L}_{1}(\underline{X}, \sigma, a)\right\}_{k} x^{k-1}} \\
p_{2}(x ; \underline{X}, \sigma)=\mathbb{I}_{[0,1]}(x) e^{-\sum_{k=1}^{3}\left\{\mathbf{L}_{2}(\underline{X}, \sigma)\right\}_{k} x^{k-1}}
\end{array}
$$

where $\mathbb{I}_{D}(x)$ is the indicator function of a given subset $D \subset \mathbb{R}$ and

$$
\mathbf{L}_{1}(\underline{X}, \sigma, a)=\arg \min _{\mathbf{L} \in \mathbb{R}^{3}} H_{1}(\mathbf{L} ; \underline{X}, \sigma, a) \quad, \quad \mathbf{L}_{2}(\underline{X}, \sigma)=\arg \min _{\mathbf{L} \in \mathbb{R}^{3}} H_{2}(\mathbf{L} ; \underline{X}, \sigma)
$$

in which

$$
\begin{gathered}
H_{1}(\mathbf{L} ; \underline{X}, \sigma, a)=\{\mathbf{L}\}_{1}+\{\mathbf{L}\}_{2} \underline{X}+\{\mathbf{L}\}_{3}\left(\sigma^{2}+\underline{X}^{2}\right)+\int_{a}^{+\infty} e^{-\sum_{k=1}^{3}\{\mathbf{L}\}_{k} x^{k-1}} d x \\
H_{2}(\mathbf{L} ; \underline{X}, \sigma)=\{\mathbf{L}\}_{1}+\{\mathbf{L}\}_{2} \underline{X}+\{\mathbf{L}\}_{3}\left(\sigma^{2}+\underline{X}^{2}\right)+\int_{0}^{1} e^{-\sum_{k=1}^{3}\{\mathbf{L}\}_{k} x^{k-1}} d x
\end{gathered}
$$




\section{Stochastic solver for the estimation of the confidence region of $S_{j}(\xi, \omega)$}

The confidence region associated with a probability level $P_{c}$ is constructed using quantiles (see for instance [12]). The method is briefly recalled. Let $F_{S_{j}(\xi, \omega)}\left(s_{j} ; \xi, \omega\right)=P\left\{S_{j}(\xi, \omega) \leq\right.$ $\left.s_{j}\right\}$ be the cumulative distribution function of the random variable $S_{j}(\xi, \omega)$. For $\left.p \in\right] 0,1[$, the $p$ th quantile of $F_{S_{j}(\xi, \omega)}$ is defined as $\zeta(p)=\inf \left\{s_{j}: F_{S_{j}(\xi, \omega)}\left(s_{j} ; \xi, \omega\right) \geq p\right\}$. Then, the upper envelope $s_{j}^{+}$and the lower envelope $s_{j}^{-}$of the confidence interval are defined by $s_{j}^{+}=\zeta\left(\left(1+P_{c}\right) / 2\right)$ and $s_{j}^{-}=\zeta\left(\left(1-P_{c}\right) / 2\right)$. Let $s_{j}^{1}=S_{j}\left(\xi, \omega ; \theta_{1}\right), \ldots, s_{j}^{n_{\mathrm{s}}}=S_{j}\left(\xi, \omega ; \theta_{n_{\mathrm{s}}}\right)$ be $n_{\mathrm{s}}$ independent realizations of $S_{j}(\xi, \omega)$. Let $\widetilde{s}_{j}^{1}=S_{j}\left(\xi, \omega ; \theta_{1}\right) \leq \ldots \leq \widetilde{s}_{j}^{n_{\mathrm{s}}}=S_{j}\left(\xi, \omega ; \theta_{n_{\mathrm{s}}}\right)$ be the order statistics associated with $s_{j}^{1}, \ldots, s_{j}^{n_{\mathrm{s}}}$. Therefore, we have the following estimation: $s_{j}^{+}=\widetilde{s}_{j}^{k^{+}}$with $k^{+}=\operatorname{int}\left(n_{\mathrm{S}}\left(1+P_{c}\right) / 2\right)$ and $s_{j}^{-}=\widetilde{s}_{j}^{k^{-}}$with $k^{-}=\operatorname{int}\left(n_{\mathrm{S}}\left(1-P_{c}\right) / 2\right)$ in which $\operatorname{int}(z)$ is the integer part of the real number $z$.

\section{References}

1. Combescure D, Pegon P. Application of the local-to-global approach to the study of infilled frame structures under seismic loading Nuclear Engineering and Design 2000 196:17-40.

2. Desceliers C, Soize C, Cambier S. Nonparametric-parametric model for random unicertainties in non-linear structural dynamics: Application to earthquake engineering. Earthquake Engineering and Structural Dynamics, 2004 33(3):315-327.

3. Durand J.F., Soize C, Gagliardini L. Structural-acoustic modeling of automotive vehicles in presence of uncertainties and experimental identification and validation Journal of the Acoustical Society of America, 2008 124(3): 1513-1525.

4. EUROCODE 8: European Committee for Standardization Design of structures for earthquake resistance. Part 3: Strengthening and repair of buildings, Draft $N^{\circ} 3,2003$.

5. Hamza S. Analyse probabiliste de la vulnérabilité sismique des btiments existants: application aux structures en portiques en béton armé $\mathrm{PhD}$ Thesis, 2007.

6. Ibrahim R.A. Structural dynamics with parameters uncertainties Applied Mechanics Reviews, 1987 40(3):309-328.

7. Jayne E. Information theory and statistical mechanics Physical Review, 1957 106(4):620630.

8. Jayne E. Information theory and statistical mechanics Physical Review, 1957 108(2):171190.

9. Kapur J.N, Kesavan H.K. Entropy optimization principles with applications Academic Press, Inc, 1992.

10. Pauley T, Priestley M.J.N. Seismic design of concrete and masonry buildings John Wiley \& Sons, 1992 .

11. Pinto P.E. Reliability methods in earthquake engineering Progress in Structural Engineering and Materials, 2001 3(1):76-85.

12. Serfling R., Approximation theorems of Mathematical Statics, Wiley, New York, 1980.

13. Shannon C. A mathematical theory of communication Bell System Technology Journal, $1948379-423$ and $623-659$

14. Soize C. Maximum entropy approach for modeling random uncertainties in transient elastodynamics Journal of the Acoustical Society of America, 2001 109(5):1979-1996.

15. Soize C. Nonlinear dynamical systems with nonparametric model of random uncertainties Uncertainties in Engineering Mechanics, 2001 1(1):1-38.

16. Soize C. Random matrix theory for modeling uncertainties in computational mechanics Computer Methods in Applied Mechanics and Engineering , 2005 194:1333-1366.

17. Soize C, Capiez-Lernout E, Ohayon R. Probabilistic model identification of uncertainties in computational models for dynamical systems and experimental validation AIAA Journal, 2008 46(11): 2955-2965.

18. Takeda T, Sozen M.A, Nielsen N.N. Reinforced concrete response to simulated earthquakes ASCE Journal of the Structural Division, 1970 96(12):2557-2573 .

19. Zienkiewicz O.C, Taylor R.L. The finite element method, volume 2 McGraw-Hill Book Company, 1991. 